\title{
WS6-C04
}

\section{Deriving P-wave Near-surface Models from Exploration Data}

\author{
E.J. van Dedem* (Shell Global Solutions International), F. Ernst (Brunei \\ Shell Petroleum Co Sdn Bhd (BSP)) \& J. Shorter (Petroleum Development \\ Oman)
}

\section{SUMMARY}

Adequate handling of the near surface is crucial for proper imaging of land data, especially for complex overburdens. Recently we introduced modal elastic inversion (Ernst 2013): approximate FWI which inverts P-guided waves to near-surface P-velocity models. We will present two case studies of modal elastic inversion of P-guided waves to obtain near-surface velocity models. The results show that this inversion approach can give realistic results not only for modern high-end densely sampled surveys, but also for sparse legacy dynamite surveys. 


\section{Introduction}

Adequate handling of the near surface is crucial for proper imaging of land data. Especially for complex overburdens with velocity inversions or high velocities near the surface, the assumptions of classical statics break down. Near-surface P-velocity models can be created from first-break picks with refraction tomography. However, first-break picking and QCing is labor intensive. Furthermore, for finite-bandwidth signals, a "first-break" is ill-defined, especially in case of shingling: When the highest first-arrival energy jumps from loop to loop, which typically occurs in areas with velocity inversions. Near-surface wavefield inversion approaches such as MASW (Xia et al., 1999) and dispersion-curve inversion (Socco and Strobbia, 2004) are gaining uptake. Ground roll, however, is sensitive mainly to S-velocity. Inversion of guided waves to P-velocity models is an area of active research (Boiero 2013). Recently we introduced modal elastic inversion (Ernst 2013): approximate FWI which inverts P-guided waves to near-surface P-velocity models. We show here examples for two very different exploration surveys: a 1990s sparse dynamite survey, and a recent high-end dense vibroseis one.

\section{Case study 1: Sparse dynamite survey}

The first example applies modal elastic inversion to a low-fold dynamite survey acquired in the 1990s in Gabon. The data has orthogonal acquisition, with $250 \mathrm{~m}$ source line spacing and $400 \mathrm{~m}$ receiver line spacing. Shot and receiver point spacing was $50 \mathrm{~m}$. Array size varied, depending on local terrain conditions, but was up to $50 \mathrm{~m}$. Figure 1 a shows a data example. The data exhibits high longwavelength and short-wavelength statics due to highly varying near-surface conditions. The acquisition parameters make surface-wave inversion impossible due to undersampling and heavy distortion by arrays. P-wave inversion from guided waves, however, is possible as these waves have velocities around $3000 \mathrm{~m} / \mathrm{s}$. Figure $1 \mathrm{~b}$ shows a depth slice through the P-wave inversion model. Clear and large velocity variations can be observed, dominated by low P-wave velocity in the river valley running north-to-south in the central part of the survey. Figure 1c shows the results of first-break tomography. The similarity of both results gives confidence in the guided-wave inversion velocity model.
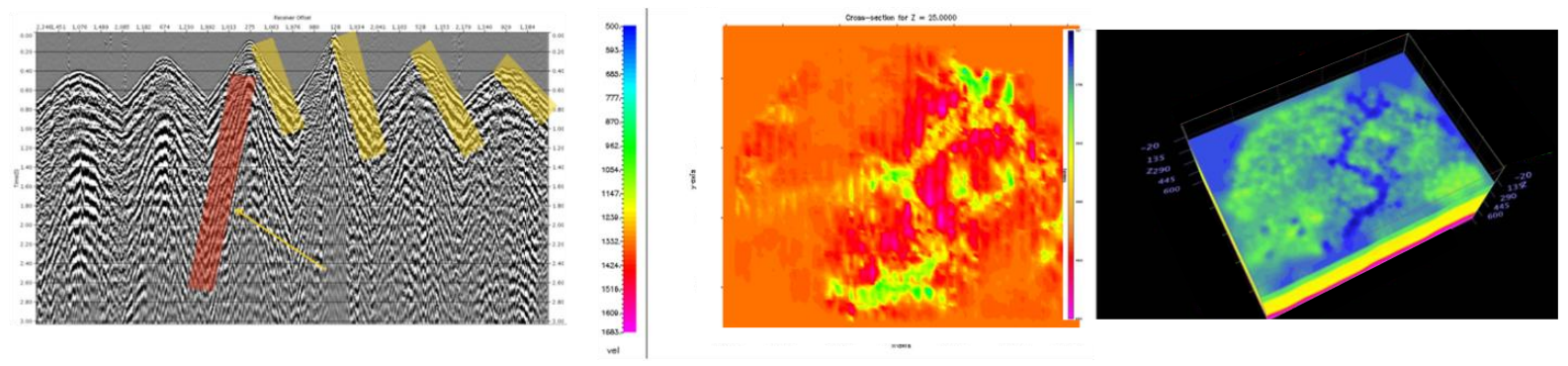

Figure 1 Left: data example: ground roll is highly aliased and attenuated (red) while guided waves are clear events (yellow). Middle: Shallow depth slice through P-wave model from modal elastic inversion; right: same for first-break-derived model.

\section{Case Study 2: Oman land data}

The Eastern Flank area in Southern Oman is notorious for its multiple-ridden data, caused by a highly reflective near-surface geology with shallow velocity inversions due to the presence of high-velocity limestones. Guided waves were inverted in the $2-15 \mathrm{~Hz}$ frequency band to a P-wave velocity model over an area of around $650 \mathrm{~km}^{2}$. Figure 2a shows a shallow P-wave velocity inversion, which is consistent with independently derived S-wave model and TDEM inversions (Figure $2 b$ and $2 c$; see also Shorter et al., 2014) and correlates with features on the stack. A map view (Figure 3) shows a clear correlation with topography and regional geology. Penetration depth of the guided waves was around $200 \mathrm{~m}$. 


\section{Amsterdam '14}
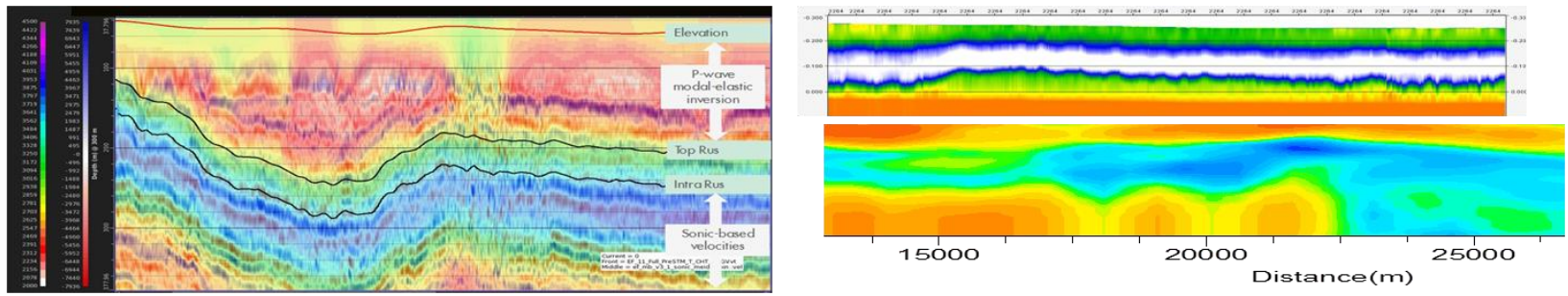

Figure 2 Left: near-surface P-wave velocity model as backdrop to stack. Right top: Results of contractor dispersion-curve inversion results (image courtesy of WesternGeco); right bottom:TDEM inversion results (image courtesy of BGP). All results show a consistent inversion in parameters.
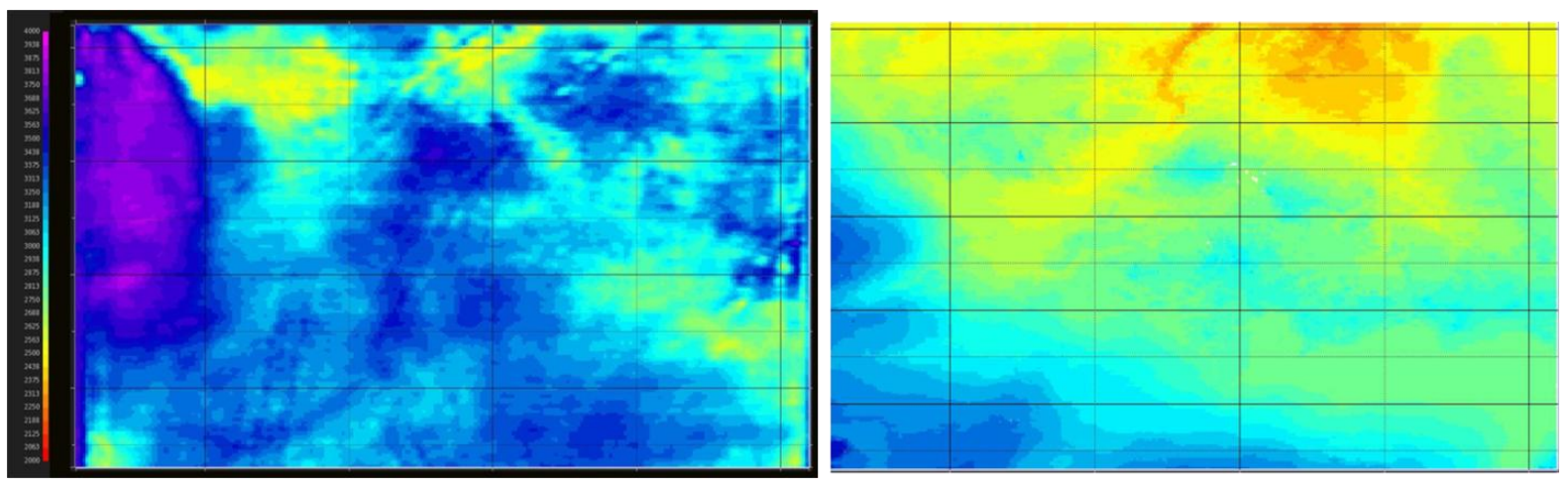

Figure 3 Map view of near-surface P-wave velocity inverted from guided waves. Right: topography (warm: low ground).

\section{Conclusion}

Accurate near-surface P-wave velocity models are rapidly gaining importance for proper imaging in areas with shallow velocity inversions or a complex overburden. We have presented two case studies of modal elastic inversion of P-guided waves to obtain near-surface velocity models. The results show that this guided-wave inversion approach can give realistic results not only for modern high-end densely sampled surveys, but also for sparse legacy dynamite surveys. Current efforts are directed towards the optimal integration of the guided-wave-based shallow model into the complete PreSDM velocity model.

\section{Acknowledgement}

The authors thank the Ministry of Oil and Gas of the Sultanate of Oman, Total Gabon and Shell for permission to publish this paper.

\section{References}

Boiero, D., C. Strobbia, L. Velasco and P. Vermeer, 2013, "Guided waves - Inversion and attenuation", EAGE $75^{\text {th }}$ Conference, London.

Ernst, F., 2013, "Modal Elastic Inversion", EAGE $75^{\text {th }}$ Conference, London.

Shorter, J., F. Ernst, H. Rynja and Q. Al-Siyabi, 2014, "Comparison of seismic and non-seismic based methods of deriving near-surface models over Eastern Flank, Oman". SEG near-surface modeling and imaging workshop, Bahrain.

Socco, L.V. and C. Strobbia, 2004, "Surface-wave method for near-surface characterization: A tutorial", Near Surface Geophysics, vol. 2, p. 165-185.

Xia, J., R.D. Miller and C.B. Park, 1999, "Estimation of the near-surface shear-wave velocity by inversion of Rayleigh waves", Geophysics, vol. 64, p. 691-700. 